\title{
A simulation study of the neutral evolution of heterozygosity and genetic distance
}

\author{
Mithu Mukherjee, \\ D. O. F. Skibinski and \\ R. D. Ward*
}

Biomedical and Physiological Research Group, School of Biological Sciences, University College of Swansea, Singleton Park, Swansea SA2 8PP, and * Environmental Biology Unit, Department of Human Sciences, University of Technology, Loughborough, Leicestershire LE11 3TU.

This paper considers a statistical test of neutral theory which can be applied to allozyme variation in natural populations. Computer simulation is used to derive the theoretical relationship between genetic distance and heterozygosity under a variety of neutral models employing stepwise mutation. It is shown that the relationship between these variables is robust to variation in the parameters of the models, and that a test of neutral theory can therefore be derived. Applications of the test in the analysis of data from natural populations are discussed in the light of the simulation results.

\section{INTRODUCTION}

One of the most important problems in evolutionary theory concerns the extent to which genetic differences within and between populations and species are caused by the action of natural selection or by random genetic drift. Although a vast amount of data on allozyme variation in natural populations has been published over the last two decades, much of it with the aim of contributing to the analysis of this problem, no decisive solution has been forthcoming. Unfortunately, this has often engendered the pessimistic views that the problem is insoluble, unimportant or badly formulated.

Practical difficulties that often arise with experimental studies of allozyme variation are that certain variables and theoretical parameters cannot be determined or measured accurately. Neutral theory has, however, a practical advantage over selection theory. Because it assumes selection coefficients are negligible there are fewer variables or parameters to be considered, and thus testable predictions may be derived more easily.

Many statistical tests of neutral theory have been devised for application to allozyme variation in natural populations. These include studies of the distribution of heterozygosity and gene frequency (Kimura and Ohta, 1971; Yamazaki and Maruyama, 1972; Wehrhahn, 1975; Nei, Fuerst and Chakraborty, 1976; Fuerst, Chakraborty and Nei, 1977; Chakraborty, Fuerst and Nei, 1980), the distribution of genetic distance (Ayala and Gilpin, 1974; Nei and Tateno, 1975), and the relationship between different statistics-for example, between the mean and variance of heterozygosity (Nei, Fuerst and Chakraborty, 1976; Nei, 1975; Gojobori, 1979), between the correlation of heterozygosity and genetic identity and between the mean and variance of genetic distance (Chakraborty, Fuerst and Nei, 1978). In most of these studies, allozyme data pooled from different sources are used in an attempt to overcome the practical difficulties referred to above. Direct attempts to test selective hypotheses have also been made using pooled data, for example, by comparing levels of variation in samples of organisms with different biotic or life history parameters (Nevo, Beiles and Ben-Shlomo, 1984).

In developing a test of neutral theory for allozyme data, there are two principal features of natural populations that can be considered. These are, first, the contemporary variation existing within populations, and second, the changes accumulated in the lineages separating populations or species. Heterozygosity is a measure of the former, genetic distance a measure of the latter. This paper is concerned with a test which analyses the relationship between these two variables (Skibinski and Ward, 1982, 1983; Ward and Skibinski, 1985). We believe the test to be potentially powerful because, unlike many tests of neutral theory (the study of Chakraborty, Fuerst and Nei 
(1978) providing an exception), it considers the covariation between these two independent aspects of natural populations. A one-dimensional test of neutral theory using genetic distance or heterozygosity alone has little power in the absence of knowledge of parameters such as mutation rate, population size and divergence time. In a twodimensional test, the requirement for such knowledge can be eliminated. The purpose of this paper is to use computer simulation to examine the relationship between heterozygosity and genetic distance under a variety of neutral models in order to provide a clear theoretical foundation for application of the test.

Many of the predictions of neutral theory have been derived for the infinite allele model of Wright (1949) rather than the more realistic stepwise mutation model originally proposed by Ohta and Kimura (1973). The latter model is rather more difficult to analyse mathematically, but fortunately the two models frequently give similar predictions. For example, at low heterozygosity the expression $H=1-1 /\left(4 N_{e} u+1\right)$ (Kimura and Crow, 1964) and $H=1-1 /\left(8 N_{e} u+1\right)^{1 / 2}$ (Ohta and Kimura, 1973) yield similar values of heterozygosity $(H)$ for given values of effective population size $\left(N_{e}\right)$ and neutral mutation rate $(u)$. Similarly, if divergence time $(t)$ is low, genetic distance $(D)$ between two species can be approximated in both models by $2 u t$. When $t$ is large, however, $2 u t$ may seriously over-estimate genetic distance in the stepwise mutation model because of the effect of backmutation. Nei and Chakraborty (1973) and Li (1976) have derived expressions for genetic identity under this model which indicate that the rate of accumulation of genetic distance slows down with increasing $t$ and that the magnitude of this effect is dependent on heterozygosity.

Nei (1972) defined genetic identity as $I_{j}=$ $\sum x_{i} y_{i} /\left(\sum x_{i}^{2} \sum y_{i}^{2}\right)$ where $x_{i}$ and $y_{i}$ are the frequencies of the $i$ th allele at the $j$ th locus in populations $X$ and $Y$ respectively. The genetic identity over all loci was defined as $I=J_{x y} /\left(J_{x} J_{y}\right)^{1 / 2}$ where $J_{x y}$, $J_{x}$ and $J_{y}$ are the arithmetic means of $\sum x_{i} y_{i}, \sum x_{i}^{2}$, and $\sum y_{i}^{2}$ over all loci. Genetic distance is then defined as $D=-\log _{e} I$, Nei (1972) noted that $I$ could also be computed as the arithmetic mean of $I_{j}$ over loci but that the genetic interpretation of this statistic was less straightforward. Hillis (1984) has argued that the mean locus identity defined as $I^{*}=\sum I_{j} / L$ (where $L$ is the number of loci) and its corresponding genetic distance $D^{*}=-\log _{e} I^{*}$ may be more appropriate measures of identity and distance than $I$ and $D$ when, as is usually observed, the extent of divergence varies between loci. In the study of Skibinski and Ward (1982), genetic distance values for enzymes were obtained after pooling data from different species comparisons. This approach led to the calculation of distance as $D^{*}$ rather than $D$. It was assumed that the theoretical predictions based on $D$ could be compared legitimately with empirical results based on $D^{*}$ for which analytical expressions are at present not available.

As well as examining the relationship between heterozygosity and genetic distance, this paper has the additional specific objectives.

(a) to compare theoretical results obtained using $D$ and $D^{*}$ as measures of genetic distance.

(b) to compare the relationship between heterozygosity and genetic distance under conditions of random mating and 100 per cent selfing. This will allow application of the test of Skibinski and Ward (1982) to allozyme variation in plants or animals showing high levels of inbreeding.

(c) To assess the effect of four factors on the relationship between heterozygosity and genetic distance and thus on the robustness of the test. These factors are, first, pooling of data from species comparisons differing in overall divergence time, second, heterogeneity of neutral mutation rates among loci contributing to individual estimates of average heterozygosity and genetic distance, third, changes in population size, and fourth, migration between diverging populations.

\section{METHOD OF COMPUTER SIMULATION}

The Fisher-Wright model of genetic population structure was used for the simulations. In each replicate simulation, for given parameter values, two populations $X$ and $Y$ were allowed to diverge over generations under the effects of mutation and random genetic drift. The stepwise mutation model (Ohta and Kimura, 1973) with one-step mutations was used with alleles numbered as integers increasing and decreasing from 100.

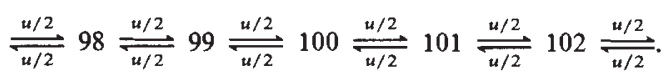

Population sizes of $N=15$ ( 30 genes), $N=30$ (60 genes), and $N=150$ (300 genes) were investigated. Results were found to be qualitatively similar with all population sizes which justifies 
extrapolation of the results to larger populations. To economise on computer time most simulations used the smallest population size which, with high mutation rates, permitted substantial genetic distance to accumulate in a relatively small number of generations.

For each replicate, genetic identity $\left(I_{j}\right)$ and heterozygosity $\left(H_{j}\right)$ were computed after a specified number $(t)$ of generations of neutral evolution. $I_{j}$ has been defined above. $H_{j}$, the average heterozygosity of populations $X$ and $Y$ at generation $t$, is defined as $\left(2-\sum x_{i}^{2}-\sum y_{i}^{2}\right) / 2$. $I$ is defined as $J_{x y} /\left(J_{x} J_{y}\right)^{1 / 2}$ where $J_{x y}, J_{x}$ and $J_{y}$ are the arithmetic means of $\sum x_{i} y_{i}, \sum x_{i}^{2}$ and $\sum y_{i}^{2}$ over all replicates and is thus analogous to $I$ as defined in the previous section. Similarly, $I^{*}$ is defined as $\sum I_{j} / L$ where $L$ is the number of replicates. In each replicate, the assignation of the labels $X$ and $Y$ to the two populations is arbitrary. Switching of the labels might thus affect the value of $I$, particularly if $L$ is small, whereas $I^{*}$ will be unaffected. The genetic distances $D$ and $D^{*}$ are defined as $-\log _{e} I$ and $-\log _{e} I^{*}$, respectively. The average heterozygosity over replicates is defined as $\bar{H}_{j}=\sum H_{j} / L$. In the present study all computed values of $D$ and $D^{*}$ are based on $L=500$ replicates. For most parameter combinations, two replicate experiments each consisting of $L=500$ replicates were carried out to allow some assessment of the effect of sampling error on heterozygosity and distance values.

In the simulations, a reliable pseudo-random number generator was used for both sampling of genes and assigning mutational events. In the random mating model, genes were picked at random with replacement to produce the next generation. In the selfing model, genes were paired in the first generation to give $N$ individuals. $N$ individuals were then picked at random with replacement and from each of these individuals two genes were picked at random with replacement to produce the $N$ individuals for the next generation.

Initial conditions of neutral equilibrium in the simulations were established in the following way. In each experiment of 500 replicates, each replicate was started with both populations $X$ and $Y$ fixed for allele 100 . After an initial period of $t^{\prime}$ generations $\bar{H}_{j}$ was measured and defined as $\bar{H}_{j}^{\prime}$. Then at the end of these $t^{\prime}$ generations, for each replicate the allele frequencies in population $Y$ were reset to be equal to the allele frequencies in population $X$. Both populations $X$ and $Y$ were then allowed to run for a further $t$ generations after which $\bar{H}_{j}$, $D$ and $D^{*}$ were measured. A value of $t^{\prime}$ was chosen, by trial and error, at which $\bar{H}_{j}^{\prime}$ was both close to its theoretical expected value, defined by the equation of Ohta and Kimura (1973), and had reached an asymptote. In this situation, it is assumed that the simulation of the $t$ generations of neutral evolution occur under conditions of neutral equilibrium.

\section{RESULTS}

Values of genetic distance $\left(D^{*}\right)$ are plotted against heterozygosity $\left(\bar{H}_{j}\right)$ for the outbreeding and inbreeding models in fig. 1 for a range of neutral mutation rates and generations of divergence. Each plotted point in all graphs represents the mean of 500 replicate simulations for a given neutral mutation rate. The range of mutation rate values are given in the legend of the figure; for the sake of clarity, mutation rate values are not indicated on the graphs. As expected, points near the origin are associated with low mutation rates and as mutation rate increases so also does genetic distance and heterozygosity. Points for duplicate experiments for given parameter combinations can usually be identified easily because of their close proximity on the graphs. Different symbols are used for different numbers of generations of divergence. In both inbreeding and outbreeding models, the relationship between the two variables is approximately linear up to about $50(3 N-4 N)$ generations and thereafter becomes increasingly asymptotic. The form of the relationship is similar or identical in both models, although for a given divergence time, the amount of genetic distance accumulated is somewhat greater in the inbreeding model. In both models, the rate of accumulation of genetic distance slows down with increasing divergence time, particularly at high heterozygosity. For comparison, fig. 1 also shows simulated relationships under the infinite allele model where, with no possibility of back-mutation, linearity is not lost at high levels of divergence. Three points are indicated as having genetic distance values of infinity. This occurs because all replicates contributing to these points have, by chance, $I_{j}$ values of zero; the expectation of genetic distance is of course less than infinity.

In the simulations with outbreeding, the effective population size $\left(N_{e}\right)$ will be equal to the actual population size $(N)$, that is 15 . In selfing populations, however, the effective population size should be $N / 2$ (Nei, 1975). Fig. 2(c) compares the results obtained in the outbreeding model with $N=15$ with those for the inbreeding model with $N=30$. It can be seen that the distance accumulated at a given heterozygosity value is now the 

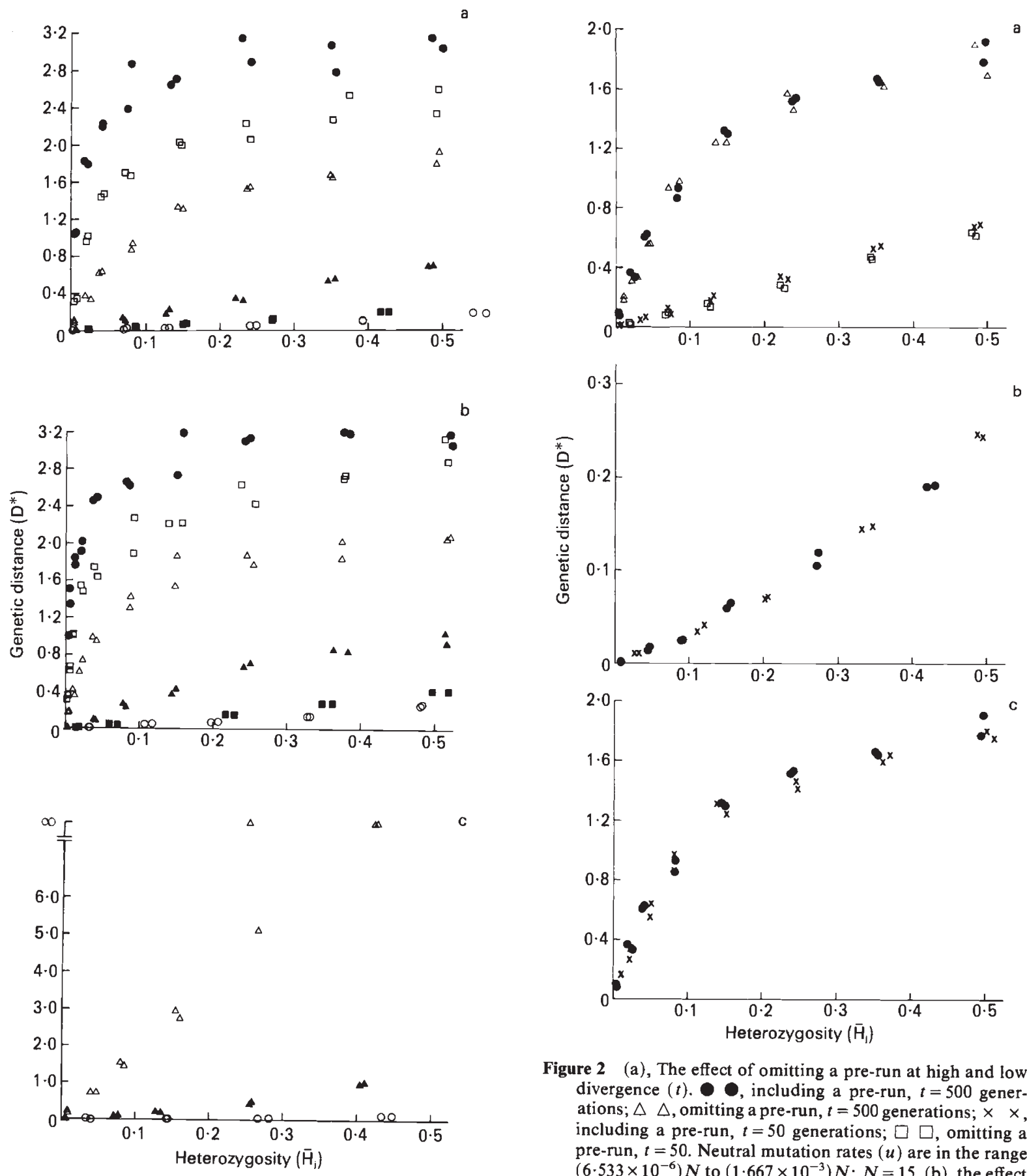

Figure 2 (a), The effect of omitting a pre-run at high and low divergence $(t)$. $\bigcirc$, including a pre-run, $t=500$ generations; $\triangle \Delta$, omitting a pre-run, $t=500$ generations; $\times \times$, including a pre-run, $t=50$ generations; $\square \square$, omitting a pre-run, $t=50$. Neutral mutation rates $(u)$ are in the range $\left(6.533 \times 10^{-6}\right) N$ to $\left(1.667 \times 10^{-3}\right) N ; N=15$. (b), the effect of simultaneously increasing population size $(N)$ and generations of divergence $(t)$. $, N=15, t=10 ; \times \times$ $N=150, t=100$. Neutral mutation rates $(u)$ are in the range $\left(6.533 \times 10^{-6}\right) N$ to $\left(1.667 \times 10^{-3}\right) N$ where $N=15$, and in the range $\left(6.533 \times 10^{-7}\right) N$ to $\left(2.083 \times 10^{-5}\right) N$ where $N=150$. (c), comparison of the outbreeding model with $N=15$ ( ) with the inbreeding model with $N=30$ $(\times \times), t=500$ generations. Neutral mutation rates $(u)$ are in the range $\left(6.533 \times 10^{-6}\right) N$ to $\left(1.667 \times 10^{-3}\right) N$ where $N=15$, and in the range $\left(3.267 \times 10^{-6}\right) N$ to $(8.333 \times$ $\left.10^{-4}\right) N$ where $N=30$. $\left(3.333 \times 10^{-3}\right) N$ in (a) and (b) and in the range $(6.533 \times$ $\left.10^{-6}\right) N$ to $\left(1.667 \times 10^{-3}\right) N$ in $(\mathrm{c}) ; N=15$. 
same, as might be expected if $N_{e}$ were identical in both models. It appears, therefore, that the theoretical relationship between genetic distance and heterozygosity is robust to this variation in the breeding system.

In fig. 2(a) the effect of omitting a pre-run is shown. These results can be regarded as simulating the recovery from a bottleneck in both populations which is so severe that the heterozygosity is reduced to zero. The form of the relationship is not affected for both $t=500$ generations and $t=50$ generations, though for the latter, the amount of genetic distance and heterozygosity accumulated for a given mutation rate is slightly less. This is expected because when a pre-run is omitted, time is required for build up of heterozygosity to equilibrium values, and during this time the rate of accumulation of genetic distance will be less than at equilibrium. After 500 generations of divergence, the effect of omitting a pre-run is negligible. This is because the pre-run represents a very small proportion of the total number of generations of evolution.

The form of the relationship would not have been the same without a pre-run if all initial heterozygosities had been set at some very high value, such as $0 \cdot 5$. In this situation, loci with low mutation rates would accumulate excess genetic distance during the fall of heterozygosities to equilibrium values.

Fig. 2(b) shows the effect of increasing population size by a factor of ten on the relationship between heterozygosity and genetic distance. Mutation rates were decreased to preserve the same range of heterozygosity values and the time of divergence was also increased by an order of magnitude to produce genetic distance values in the same range. A longer pre-run was also used in order to reach initial equilibrium values. The relationship is identical for the two population sizes. It is therefore reasonable to assume that the form of the relationship at a given level of divergence is independent of population size. Thus conclusions obtained with the smaller population may be reasonably extrapolated to larger populations.

In fig. 3(a) the two measures of genetic distance $D$ and $D^{*}$ are compared. $D$ is slightly greater than $D^{*}$, particularly at high divergence, but the difference is negligible. Fortunately, this suggests that theoretical predictions derived for the statistic $D$ may be tested using experimental genetic distance estimates expressed as either $D^{*}$ or $D$.

In fig. 3(b) and 3(c) analytically derived and simulated values of the statistics are compared for the outbreeding model. First, in fig. 3(b) simulated heterozygosity values are compared with values
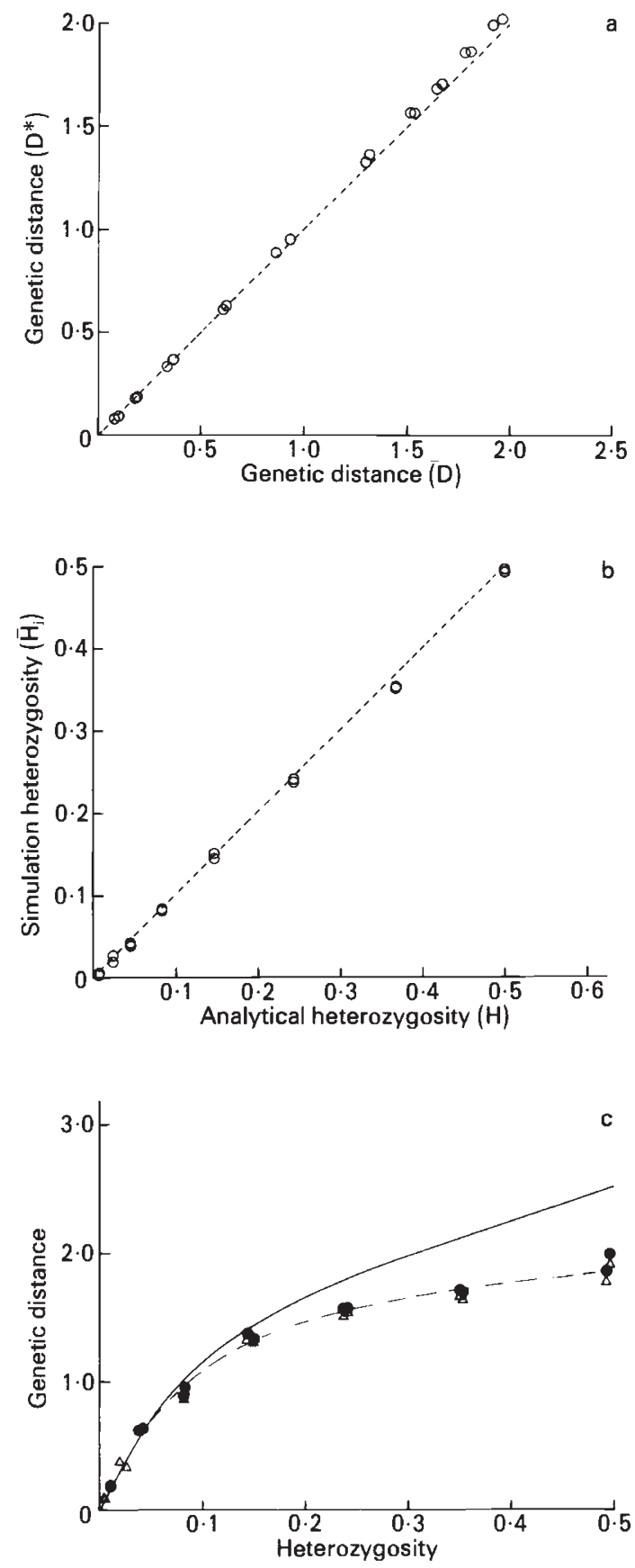

Figure 3 (a), Comparison of simulated values of genetic distance $D$ with those of $D^{*}$. - - - line of $45^{\circ}$. (b), comparison of analytically derived and simulated values of heterozygosity. - - - line of $45^{\circ}$. (c), comparison of analytically derived and simulated relationships between genetic distance and heterozygosity. for genetic distance, $D ; \triangle \triangle$, simulated values for genetic distance, $D^{*} ;-\ldots$, Nei's equation (2); _- —, Li's equation (3); $t=500$ generations in all cases. Neutral mutation rates $(u)$ are in the range $\left(6.533 \times 10^{-6}\right) N$ to $(1.667 \times$ $\left.10^{-3}\right) N ; N=15$ in all cases. 
from the equation

$$
H=1-1 /\left(1+8 N_{e} u\right)^{1 / 2}
$$

for the stepwise mutation model (Ohta and Kimura, 1973). $N_{e}$ is equal to 15 and the values of $u$ chosen are identical to those for the simulations. There is close agreement between analytical and simulated values which, in this instance, provides a check against the reliability of the computer program and randomisation procedure. In fig. 3(c) analytically derived and simulated relationships between genetic distance and heterozygosity are compared. The formula of Nei and Chakraborty (1973) can be simplified to

$$
I=e^{-2 u t} \sum_{r=0}^{\infty}(u t)^{2 r} /(r !)^{2} \quad(\mathrm{Nei}, 1978)
$$

and $D=-\log _{e} I$.

Li's (1976) formula is

$$
D=2 u t-\log _{e} \sum_{i=-\infty}^{\infty} Z_{i}^{|i|} I_{i}(2 u t)
$$

where

$$
I_{i}(2 u t)=\sum_{s=0}^{\infty}(u t)^{2 s+i} / s !(i+s) !
$$

and

$$
Z_{1}=\left(1+4 N_{e} u-\left(1+8 N_{e} u\right)^{1 / 2}\right) / 4 N_{e} u \quad(\mathrm{Li}, 1976) .
$$

Computer programs were used to evaluate equations (2) and (3) for different values of $u$ with $N_{e}=15$ and $t=500$. The values of $D$ obtained are plotted against $H$ from equation (1) in fig. 3(c). The simulated results fit those for equation ( 3 ) well, but there is a poor fit with equation (2) except at low heterozygosity. Chakraborty and Nei (1977) anticipated that equation (2) may not be applicable at high heterozygosity. The simulated results for $D^{*}$ also fit equation (3) well.

In fig. 4(a) the effect of varying mutation rate $(u)$ between replicates within each experiment of 500 replicates is shown. This is analogous to the situation where the mutation rate of loci scored for a particular enzyme varies about some mean value, and that differences in mean occur between different enzymes. It is assumed that $u$ varies according to a gamma distribution (Nei, Fuerst and Chakraborty, 1976; Nei, Chakraborty and Fuerst, 1976). The probability density function

$$
p(u)=u^{G-1} e^{-u / H} / H^{G}(G-1) !
$$

was used, where $G$ and $H$ are parameters of the distribution. With $G=1$, the distribution is
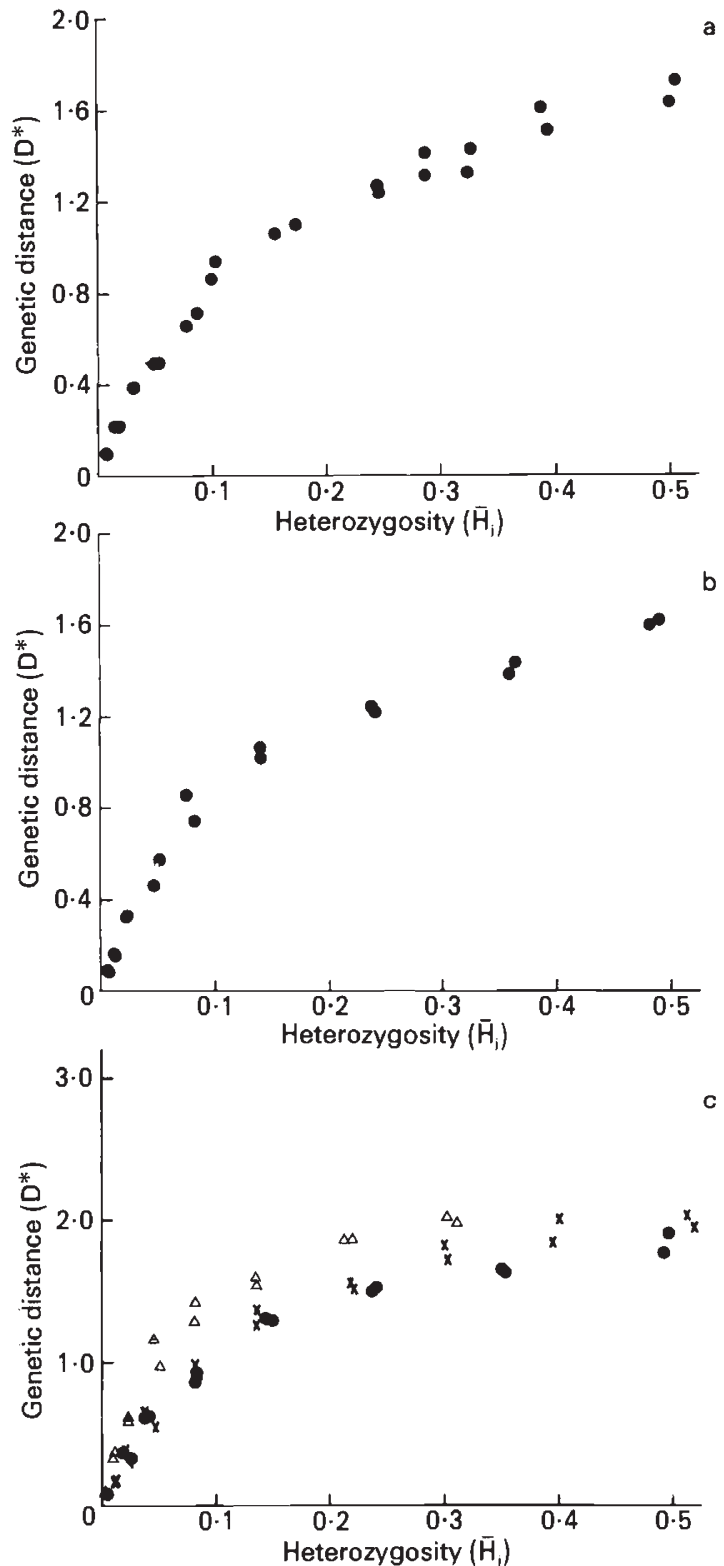

Figure 4 (a), The effect of varying mutation rate $(u)$, where $u$ is chosen at random from a gamma distribution and mean mutation rates $(\bar{u})$ are in the range $\left(7.267 \times 10^{-6}\right) N$ to $\left(2.920 \times 10^{-3}\right) N ; N=15$. (b), the effect of varying divergence time $(t)$ for each of the 500 replicate pairs of populations. Mean divergence time is approximately 500 generations, and neutral mutation rates $(u)$ are in the range $\left(6.533 \times 10^{-6}\right) N$ to $\left(1.667 \times 10^{-3}\right) N ; N=15$. (c), the effect of a difference in size $(N)$ between the two populations. $\triangle \triangle$, populations differ in size, $N=15$ for $X$ and $N=2$ for $Y, t=500$ generations. Neutral mutation rates $(u)$ are in the range $\left(9.8 \times 10^{-5}\right)$ to $\left(2.5 \times 10^{-2}\right) ; 0$, no difference in size, $t=500$ generations. Neutral mutation rates $(u)$ are in the range $\left(9.8 \times 10^{-5}\right)$ to $\left(2.5 \times 10^{-2}\right) ; \times \times$, populations differ in size, $N=15$ for $X$ and $N=2$ for $Y, t=250$ generations. Neutral mutation rates $(u)$ are in the range $\left(3.91 \times 10^{-4}\right)$ to 0.1 . 
skewed, with attenuation to the right as expected for mutation rate. For each experiment, a value of $H$ was chosen, and values of $u$ picked at random from the distribution. $H$ was varied to simulate variation in mean mutation rate between proteins.

A divergence time of $t=500$ generations was used. If fig. 4(a) is compared with fig. 1(a) for $t=500$ generations where variance of $u$ is of course zero within experiments, similar asymptotic relationships are observed. Thus the variation in mutation rate has little or no effect on the relationship between heterozygosity and genetic distance.

In fig. 4(b) the effect of varying divergence time $(t)$ between replicates within each experiment of 500 replicates is shown. This is analogous to the situation where the loci scored for a particular enzyme come from different species comparisons which differ in overall genetic distance and divergence time. For simulation, $t$ was varied at random between the limits 5 and 995 such that the average of $t$ was approximately 500 generations for each experiment and thus comparable with the results of fig. 1(a) where $t=500$ generations. It is clear from comparisons of the two figures that random variation in $t$ has a negligible effect on the relationship between genetic distance and heterozygosity.

Up to this point, it has been assumed that the two populations $X$ and $Y$ remain equal in size, and do not change in size throughout the period of divergence. Some simulations have therefore been carried out to assess the effects of variations in population size.

In fig. $4(\mathrm{c})$, the effect of the two populations differing in size at the beginning of the period of divergence is shown. Values of $N=15$ and $N=2$ were given to populations $X$ and $Y$, respectively, and a pre-run of 15 generations was used with $t=500$ generations. For comparison, fig. 4(c) also shows the results obtained with the populations equal in size $(N=15)$. As expected, mean heterozygosity is reduced for a given mutation rate, and genetic distance is increased for a given heterozygosity value, when population $Y$ is reduced in size. The familiar asymptotic relationship is however retained. The effect of a smaller number of generations of divergence with the populations differing in size is also shown. The value of $t$ was chosen, by trial and error, so that the relationship obtained fits closely to that for $t=500$ generations with the populations identical in size. The form of the relationship appears to be hardly affected by a difference in size between populations.

In fig. 5(a) and 5(b) the effects of changes in population size during divergence on the relation- ship between heterozygosity and genetic distance are shown. In fig. 5(a) populations $X$ and $Y$ differ in size initially with $N=15$ and $N=2$, respectively. Population $X$ is allowed to evolve for 400 generations with $N=15$. Then for a final 100 generations it is reduced in size to $N=2$. Population $Y$ is allowed to evolve for 100 generations with $N=2$. Then for a final 400 generations the size is increased to $N=15$. Thus one population undergoes a contraction in size, the other undergoes an expansion. The changes in size in the two populations occur at different times. For comparison, results for $t=500$ generations with the populations equal in size $(N=15)$ throughout the period of divergence are shown. Fig. 5(a) also shows the effect of a smaller number of generations of evolution with population size changes. Population $X$ is allowed to evolve for 200 generations with $N=$ 15 , then for 50 generations with $N=2$. Population $Y$ evolves for 50 generations with $N=2$, then for 200 generations with $N=15$. The value of $t$ was chosen so that the resulting relationship fits as closely as possible to that obtained for $t=500$ generations with populations identical in size $(N=$ 15) throughout the period of divergence. Since heterozygosity is reduced by the smaller population sizes, higher mutation rates were used to give the same range of heterozygosity values. The form of the relationship is hardly affected by the arbitrary changes in population size.

In fig. 5(b) both populations $X$ and $Y$ are allowed to evolve for 400 generations with $N=15$. Then for a final 100 generations the size of both populations is reduced to $N=2$. Results for divergence for $t=500$ generations with $N=15$ are also shown for comparison. As expected, heterozygosity is reduced considerably by the contraction in size. Also, at a given heterezygosity value a substantial increase in the amount of genetic distance accumulated occurs in the presence of the contraction. This can be attributed to the rapid fixation which occurs during a contraction in size or bottleneck as a result of strong genetic drift (Chakraborty and Nei, 1977).

Fig. 5(b) also shows the effect of a smaller number of generations of evolution with a contraction in size. Both populations $X$ and $Y$ evolve for 40 generations with $N=15$ followed by 10 generations with $N=2$. These values have been chosen, by trial and error, so that the resulting relationship fits as closely as possible to that obtained for $t=$ 500 generations without the contraction in size. As in fig. 5(a), rather higher mutation rates had to be used to give a comparable range of heterozygosity values. The form of the relationship between 

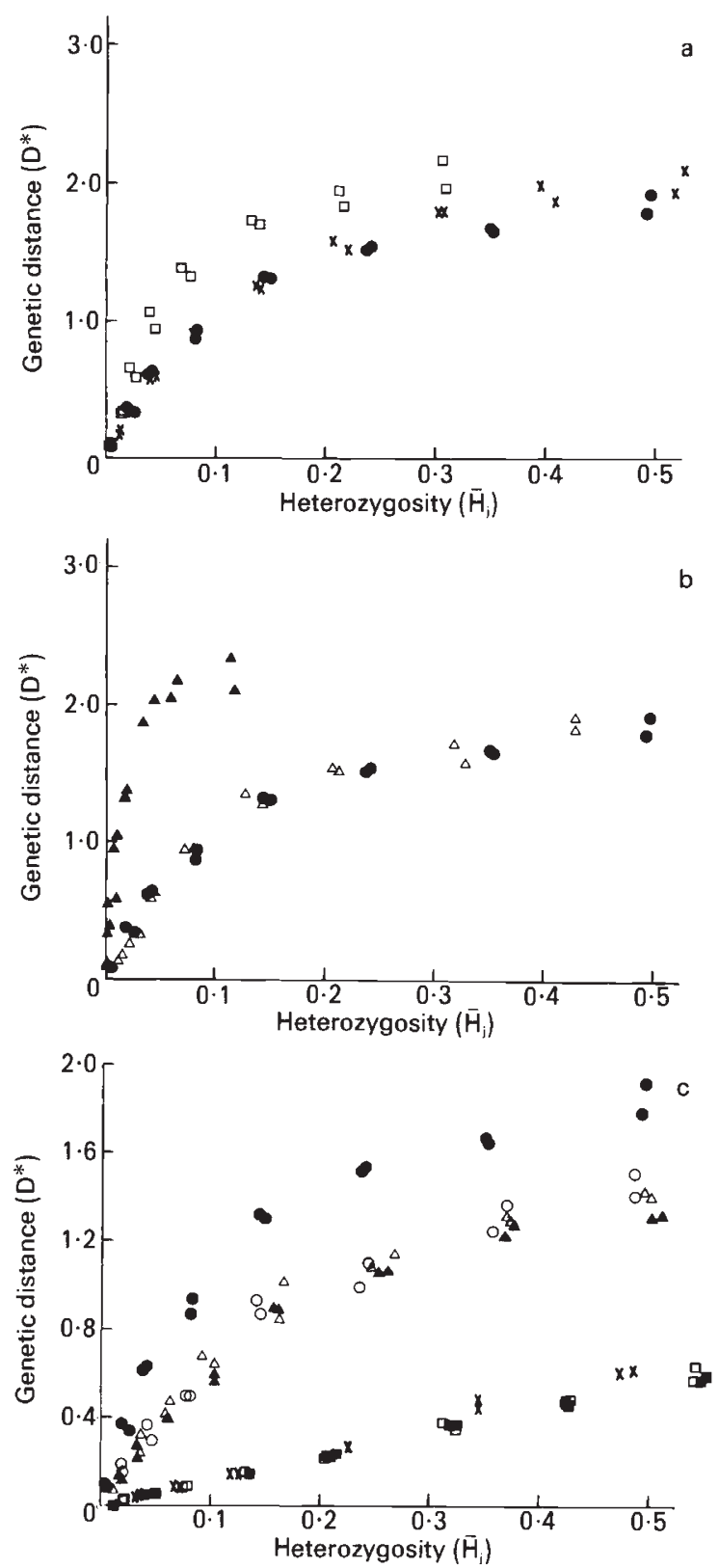

Figure 5 (a), The effect of changes in population size during divergence. $\square \square$, size of $X$ is reduced from $N=15$ to $N=2$ after 400 of a total of $t=500$ generations, size of $Y$ is increased from $N=2$ to $N=15$ after 100 of a total of $t=500$ generations. Neutral mutation rates $(u)$ are in the range $\left(9.8 \times 10^{-5}\right)$ to $\left(2.5 \times 10^{-2}\right) ; \bigcirc$, absence of size changes, $t=500$ generations. Neutral mutation rates $(u)$ are in the range $\left(9.8 \times 10^{-5}\right)$ to $\left(2.5 \times 10^{-2}\right) ; \times \times$, size of $X$ is reduced after 200 of a total of $t=250$ generations, size of $Y$ is increased after 50 of a total of $t=250$ generations. Neutral mutation rates $(u)$ are in the range $(3.91 \times$ $10^{-4}$ ) to $0 \cdot 1$. (b), the effect of a contraction in size. $\boldsymbol{\Delta} \Delta$, presence of a contraction, $t=500$ generations. Neutral mutation rates $(u)$ are in the range $\left(9.8 \times 10^{-5}\right)$ to $(2.5 \times$ $\left.10^{-2}\right)$, absence of a contraction, $t=500$ generations. genetic distance and heterozygosity is almost identical with and without the contraction in population sizes.

In fig. 5(c) the effect of migration is shown. Migration rates of $m=0.001$ and $m=0.01$ were used. These correspond to one gamete migrating every 33 and every three generations respectively. Migration occurs between both populations $X$ and $Y$. Increasing the migration rate from zero to $m=$ 0.001 , then to $m=0.01$ (with $t=500$ generations in each case) has the expected effect of reducing the amount of genetic distance accumulated. The simulations of $t=500$ and $t=2000$ generations for $m=0.001$ do not differ in the amount of genetic distance accumulated. (A similar result is obtained for $m=0.01$.) This suggests an equilibrium at which the accumulation of genetic distance by drift is balanced exactly by migration between populations. The form of the relationship between heterozygosity and genetic distance is hardly affected by migration. Thus it can be seen from fig. 5(c) that with zero migration ( $t=250$ and $t=40$ generations) relationships can be found which fit well those obtained when migration occurs.

\section{DISCUSSION}

The aim of examining the theoretical relationship between heterozygosity and genetic distance is to provide a test of neutral theory which can be applied to data from natural populations. For this, sets of loci differing in evolutionary rate, and compared in related populations or species, are needed to provide estimates of heterozygosity and genetic distance. With allozyme data, the sets of loci may be represented by different enzymes, for which there is evidence of differences in evolutionary rate (Sarich, 1977; Skibinski and Ward, 1982), and which under the neutral hypothesis would imply differences in neutral mutation rate. A practical difficulty is that two species will provide little data, usually one and seldom more than two or three estimates of locus heterozygosity and identity for a given enzyme. Skibinski and Ward (1982) and

Figure 5 (continued)

Neutral mutation rates, $u$, are in the range $\left(9.8 \times 10^{-5}\right)$ to $\left(2.5 \times 10^{-2}\right) ; \triangle \triangle$, presence of a contraction, $t=50$ generations. Neutral mutation rates, $u$, are in the range $(9.8 \times$ $\left.10^{-5}\right)$ to $\left(2 \times 10^{-1}\right)$. (c), the effect of migration. $\boldsymbol{\Delta} \boldsymbol{\Delta}$, migration, $m=0.001, t=500 ; \Delta \Delta$, migration, $m=0.001, t=$ 2000; $\square$, migration, $m=0 \cdot 01, t=500 ; \square \square$, migration, $m=0.01, t=2000 ; \bigcirc$, no migration, $t=500 ; \bigcirc \bigcirc$, no migration, $t=250 ; \times \times$, no migration, $t=40$. Neutral mutation rates, $u$, are in the range $\left(9.8 \times 10^{-5}\right)$ to $(2.5 \times$ $\left.10^{-2}\right) ; N=15$. 
Ward and Skibinski (1985) have attempted to overcome this problem by pooling allozyme data from a large number of published species comparisons and attempting to correct statistically for differences among individual species comparisons in overall distance and divergence time. This enables genetic distance to be plotted against average heterozygosity over a sample of proteins. Even though average divergence time for the species contributing data is unknown, under neutral theory a relationship of the type shown in fig. 1(a) should fit the data. Evidence against a neutral interpretation could be provided in two ways. First, if a line of best fit to the observed data was clearly not consistent with any monotonic relationship of the type shown in fig. 1, or second, if the scatter of points about a line of best fit was greater than could be attributed to drift and sampling errors. In particular, an observation that proteins with similar heterozygosities have widely different genetic distances, or that proteins with widely different heterozygosities have similar genetic distances could not easily be accounted for by neutral theory.

Skibinski and Ward (1982) interpreted an observed positive intercept on the genetic distance axis in the regression of protein genetic distance on heterozygosity as evidence against neutral theory. In that study, it was assumed that the theoretical relationship between the two variables based on approximate analytical equations is linear and not, as reported here, asymptotic. Chakraborty and Hedrick (1983) and Chakraborty (1984) objected to the interpretation primarily on statistical grounds though we are not convinced by their criticisms (Skibinski and Ward, 1983). It appears, however, that with the asymptotic relationship derived by computer simulation as in fig. 1, a linear regression analysis would produce a positive intercept on the genetic distance axis at high divergence. For this reason, the results of Skibinski and Ward (1982) appear to be consistent with neutral theory.

In computer simulation it is not possible to make an exhaustive study of the effect of all possible parameter values and combinations. We do feel, however, that we have considered a sufficient variety of neutral models to support the suggestion that the predictions of neutral theory in relation to the test are fairly robust to variation in underlying parameters. Thus, application of the test does not require assumptions about the values of effective population size, neutral mutation rates or divergence times. The relationships obtained are identical in the inbreeding and outbreeding models, suggesting that pooling of experimental data from species with different breeding systems or population structures would be legitimate. The form of the relationship is not affected by contractions, expansions or bottlenecks in population size, suggesting that the test can be applied to data from species with a history of such changes, which contradicts analytical conclusions of Chakraborty and Hedrick (1982) and Chakraborty (1984). Neither is it affected by migration between diverging populations. Heterogeneity of mutation rates between loci within categories such as particular enzymes (i.e., between replicates within experiments in the simulations) does not markedly alter the relationship. Similarly, heterogeneity of divergence time between single locus estimates of identity and heterozygosity has little effect on the relationship provided that the mean divergence time is similar for different categories.

Estimates of genetic distance based on allozyme data are often used in experimental studies to provide estimates of divergence time. The results reported here confirm not only that such estimates, obtained through linear extrapolation, are inaccurate at high distance values, but also that account should be taken of the distribution of proteins differing in mutation rates contributing to an estimate. In principle, an alternative to the use of genetic distance as a measure of evolutionary rate would be to construct a phylogeny for each study of allozyme variation and add up for each enzyme the total number of changes along branches in all phylogenies where that enzyme is scored. With this method there would be the problem of uncertainty of choice between alternative phylogenies for particular data, and also, with a large number of studies, the cost of computer time might be prohibitive.

The main problem in applying the test with allozymes is the necessity of pooling data differing in overall divergence time. In an analysis of the relationship between heterozygosity and genetic distance for enzyme and blood group racial variation in man, Chakraborty (1984) has bypassed this problem by simply regressing locus genetic distance on locus heterozygosity. In general we are not in favour of this application of the test. There are large errors associated with single locus heterozygosity and distance and the distribution of distance is greatly dependent on heterozygosity, being actually bimodal at low heterozygosity. Moreover, this test cannot be applied to any set of data which contains loci fixed non-identically in different species or populations because, in this situation, locus distance would be infinity. 
Chakraborty shows that for the enzyme loci, a zero regression intercept is obtained suggesting neutral evolution. This is consistent with our observations on inter- and intra-population variation in vertebrates and invertebrates (Ward and Skibinski, 1985) and plants (Mukherjee, Skibinski and Ward, unpublished data), where for low divergences a zero regression intercept is also obtained. For the blood group loci, heterozygosity and distance values were rather higher and low regression coefficients and higher intercepts on the distance axis were obtained. For blood group loci, Chakraborty thus supported an interpretation involving selection. His theoretical analysis does however assume the infinite allele model, which, as he admits, may not be suitable for studying genetic variation at blood group loci. The blood group results can, however, be explained adequately by the neutral stepwise mutation model because of the asymptotic relationship between distance and heterozygosity that this model generates. By contrast, it is seen here that the infinite allele model generates a roughly linear relationship between the variables even up to high divergence.

In the future, problems associated with pooling data may be overcome by using data from studies where cloned DNA is sequenced from related species or populations. In this situation, different categories could be represented by, for example, different codon positions or sequences from coding and noncoding regions. Because of the large number of sites which can be scored, it should be possible with DNA to obtain sufficient data for different categories to apply the test using small numbers of individual organisms from just two diverged populations or species.

An unanswered question concerns the extent to which different selective models produce results similar or different to those observed for strictly neutral theory. It seems that this question cannot easily be answered using analytical methods, or by the rapid diffusion approximation method of Griffiths and Li (1983) for simulating the distribution of shared alleles between diverging populations under neutral theory. We are at present attempting to answer this question using modifications of the programs employed in this study.

Acknowledgements We wish to thank Dr Peter Donnelly of the Department of Mathematics and Derwen Evans of the Computer Centre, University College of Swansea, for help and advice.

\section{REFERENCES}

AYALA, F. J. AND GILPIN, M. E. 1974. Gene frequency comparisons between taxa: support for the natural selection of protein polymorphisms. Proceedings of the National Academy of Sciences, 71, 4847-4849.

CHAKRABORTY, R. 1984. Relationship between heterozygosity and genetic distance in the three major races of man. American Journal of Physical Anthropology, 65, 249-258.

CHAKRABORTY, R., FUERST, P. A. AND NEI, M. 1978. Statistical studies on protein polymorphism in natural populations. II. Gene differentiation between populations. Genetics, 88 , 367-390.

CHAKRABORTY, R. AND NEI, M. 1977. Bottleneck effects on average heterozygosity and genetic distance with the stepwise mutation model. Evolution, 31, 347-356.

CHAKRABORTY, R., FUERST, P. A. AND NEI, M. 1980. Statistical studies on protein polymorphism in natural populations. III. Distribution of allele frequencies and the number of alleles per locus. Genetics, 94, 1039-1063.

CHAKRABORTY, R. AND HEDRICK, P. W. 1983. Heterozygosity and genetic distance of proteins. Nature, 304, 755-756.

FUERST, P. A., CHAKRABORTY, R. AND NEI, M. 1977. Statistical studies on protein polymorphism in natural populations. I. Distribution of single locus heterozygosity. Genetics, 86 , 455-483.

GOJOBORI, T. 1979. Ph.D. Thesis, Kyushu University, Fukuoka, Japan.

GRIFFITHS, R. C. AND LI, W. H. 1983. Simulating allele frequencies in a population and the genetic differentiation of populations under mutation pressure. Theoretical Population Biology, 23, 19-33.

HILliS, D. M. 1984. Misuse and modification of Nei's genetic distance. Systematic Zoology, 33, 238-240.

KIMURA, M. AND CROW, J. F. 1964. The number of alleles that can be maintained in a finite population. Genetics, 49, 725-738.

KIMURA, M. AND OHTA, T. 1971. Protein polymorphism as a phase of molecular evolution. Nature, 229, 467-469.

LI, W. H. 1976. Electrophoretic identity of proteins in a finite population and genetic distance between taxa. Genetical Research, 28, 119-127.

NEI, M. 1972. Genetic distance between populations. American Naturalist, 106, 283-292.

NEI, M. 1975. Molecular Population Genetics and Evolution, North-Holland Publication Co., Amsterdam.

NEI, M. 1978. The theory of genetic distance and evolution of human races. Japanese Journal of Human Genetics, 23, 341-369.

NEI, M. AND CHAKRABORTY, R. 1973. Genetic distance and electrophoretic identity of proteins between taxa. Journal of Molecular Evolution, 2, 323-328.

NEI, M., CHAKRABORTY, R. AND FUERST, P. A. 1976. Infinite allele model with varying mutation rate. Proceedings of the National Academy of Sciences, 73, 4164-4168.

NEI, M., FUERST, P. A. AND CHAKRABORTY, R. 1976. Testing the neutral mutation hypothesis by distribution of single locus heterozygosity. Nature, 262, 491-493.

NEI, M. AND TATENO, Y. 1975. Interlocus variation of genetic distance and the neutral mutation theory. Proceedings of the National Academy of Sciences, 72, 2758-2760.

NeVo, E., BEIles, A. AND BEN-SHlomo, R. 1984. The evolutionary significance of genetic diversity: ecological, demographic and life history correlates. Lecture Notes in Biomathematics, 53, 13-213. 
OHTA, T. AND KIMURA, M. 1973. A model of mutation appropriate to estimate the number of electrophoretically detectable alleles in a finite population. Genetical Research, 22, 201-204.

SARICH, V. M. 1977. Rates, sample sizes, and the neutrality hypothesis for electrophoresis in evolutionary studies. Nature, 265, 24-28.

SKIBINSKI, D. O. F. AND WARD, R. D. 1982. Correlations between heterozygosity and evolutionary rate of proteins. Nature, 298, 490-492.

SKIBINSKI, D. O. F. AND WARD, R. D. 1983. Heterozygosity and genetic distance of proteins-reply to Chakraborty and Hedrick. Nature, 304, 755-756.
WARD, R. D. AND SKIBINSKI, D. O. F. 1985. Observed relationships between protein heterozygosity and protein genetic distance and comparisons with neutral expectations. Genetical Research, 45, 315-340.

WEHRHAHN, C. F. 1975. The evolution of selectivity similar electrophoretically detectable alleles in finite natural populations. Genetics, 80, 375-394.

WRIGHT, S. 1949. Genetics of populations. Encyclopaedia Britannica, 14 ed. 10. 111, 111A-D, 112.

YAMAZAKI, T. AND MARUYAMA, T. 1972. Evidence for the neutral hypothesis of protein polymorphism. Science, 178, 56-58. 